\title{
The Effects of the EMDR Group Traumatic Episode Protocol With Cancer Survivors
}

\author{
Amanda Karen Patricia Roberts \\ Private Practice, Amherst, Massachusetts
}

\begin{abstract}
The purpose of this pre-experimental case study was to explore the efficacy and safety of the Eye Movement Desensitization and Reprocessing (EMDR) Group Traumatic Episode Protocol (G-TEP) in the psychological treatment of cancer survivors and its potential effects on posttraumatic stress, anxiety, and depressive symptoms. Participants $(N=35)$ were patients with various types of cancer, in different stages, initial or recurring, with diagnosis or oncology treatment received within the past year. Following an individual psychoeducational intake session, participants received two 90-minute EMDR G-TEP sessions, administered on consecutive days. They were randomly assigned to a treatment group or a delayed treatment group. Assessments were administered at pre, post, and follow-up using the Short PostTraumatic Stress Disorder Interview (SPRINT), State-Trait Anxiety Inventory (STAI), and Beck Depression Inventory (BDI-II). Repeated measures comparisons of PTSD symptoms, anxiety, and depression revealed significant differences between pretest and posttest, with most results maintained at follow-up. Pre-follow-up effect sizes showed medium effects. These promising results suggest the value in providing a lengthier course of treatment. They support the need for research with large sample, randomized clinical trials to examine the viability of providing EMDR G-TEP in the psychological treatment of cancer survivors. No serious adverse effects were reported and we conclude that the EMDR G-TEP may be effective and safe in the psychological treatment of an oncology population.
\end{abstract}

Keywords: cancer; eye movement desensitization and reprocessing; G-TEP

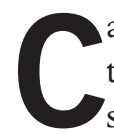
ancer can be deadly. World Health Organization (WHO, 2017) determined that cancer is the second leading cause of morbidity and mortality worldwide, with approximately 14 million new cases in 2012 and 8.8 million deaths in 2015. Globally, nearly one in six deaths are due to cancer. The WHO has estimated a projected $70 \%$ increase in diagnosed cases over the next two decades. The economic impact of cancer is large and increasing. The annual economic cost of cancer in the United States alone for 2020 is estimated at approximately $\$ 157.77$ billion, a 39\% increase from 2010 (Mariotto, Robin Yabroff, Shao, Feuer, \& Brown, 2011; WHO, 2017). Therefore, the need for effective psychological interventions is self-evident.

\section{Psycho-Oncology as an Emerging Field}

\section{Unique Challenges Cancer Survivors Face}

Faced with medical, life-altering, and often life-threatening circumstances, patients living with cancer often feel overwhelmed by the myriad of challenges they face. Not only have they endured the shock of the diagnosis, but they are often confronted by physical symptoms of fatigue, insomnia, nausea, and pain, along with all of the emotional symptoms of worry, isolation, hopelessness, grief, and fear. Many face the brutal onslaught of medical treatments that include invasive surgery, chemotherapy, and radiation. A cancer diagnosis can have a major impact on family relationships, friendships, and socioeconomic occupational functioning (Naughton \& Weaver, 2014).

People with cancer face the reality that it is often a chronic condition with periods of remission and then relapse. Survivors may never again feel the same level of physical and emotional safety that they once enjoyed. Without a posttrauma safety period, survivors may not readily reach a stage of resolution where long-term memories are consolidated (Jarero, Artigas, Uribe, \& García, 2016). In such cases, memory networks can be understood to remain in an excitatory state, creating cumulative trauma exposure without respite (Jarero, 
Artigas, \& Luber, 2011; Jarero, Amaya, Givaudan, \& Miranda, 2013).

\section{Psychosocial Support Groups}

Empirical evidence has established both a theoretical and causal impact of relationships on health. Social isolation is a major risk factor for mortality (House, Landis, \& Umberson, 1988). The global cancer epidemic had received little attention from the mental health community up until the past three decades with the introduction of psychosocial support groups. Psycho-oncology is now an emerging field that addresses a patient's psychological discomfort arising from the traumatizing sequelae of the disease (Morasso et al., 2002). A study on the effects of laughter and humor in a group format on the quality of life in cancer patients revealed statistically significant reductions in depression, anxiety, and physical pain (Roberts, 1993). Giese-Davis et al. (2016) conducted a survey among 292 patients who had participated in different types of cancer support groups, to examine the strengths and weaknesses of therapist-led and nontherapist-led, supportive-expressive therapy, and wellness community groups. They concluded that the different types of supportive groups addressed different topics and that not all groups should be expected to reduce traumatic distress. These studies illustrate that psychosocial supports for cancer survivors should be matched with the individual's unique requirements.

\section{Social Support}

Evidence of the positive effects of social support on health is clear. A review of 16 studies summarizing knowledge on psychosocial support recommended that support be offered to cancer survivors continuously throughout the cancer trajectory (Hoeck, Ledderer, \& Hansen, 2015). Psychosocial support groups have improved the quality of cancer survivors' lives (Spiegel, Bloom, Kraemer, \& Gottheil, 1989). Since the Stanford study, cancer support groups have become ubiquitous; however, these groups are not suitable for all cancer survivors. Many cancer survivors do not attend groups due to social anxiety and the fear of exposure to the traumatic cancer narratives of their peers (Galinsky $\&$ Schopler, 1994). Talk therapy groups can be triggering for survivors, and therefore, in some cases, counterproductive.

\section{EMDR Therapy}

EMDR therapy was endorsed by the WHO (2013) as a front-line treatment for psychological trauma in its clinical protocols and guidelines. The model on which EMDR is based, Adaptive Information Processing (AIP), posits that much of psychopathology is due to the maladaptive encoding of and/or incomplete processing of traumatic or disturbing adverse life experiences (Shapiro, 2001, 2004, 2012, 2013b, 2018). Potential mechanisms of change underlying the AIP theory have been fully explored and discussed (Solomon \& Shapiro, 2008). This is assumed to impair the patient's ability to integrate these experiences in an adaptive manner. The eight-phase, three-pronged process of EMDR aims to facilitate the resumption of normal information processing and integration. During EMDR therapy, clients focus on memory-related material while engaging in brief sets of eye movement. This treatment approach, which targets past experiences, current triggers, and future potential challenges, results in the alleviation of presenting symptoms, a decrease or elimination of distress from the disturbing memory, improved view of the self, relief from bodily disturbance, and resolution of present and future anticipated triggers (EMDR International Association, 2012).

\section{EMDR Treatment of Cancer-Related Traumatic Stressors}

EMDR Individual Treatment. The diagnosis and treatment of cancer are uniquely traumatic stressors (Andrykowski \& Kangas, 2010). The EMDR community has recently begun to focus on the emotional suffering that cancer produces and on interventions to alleviate distress (EMDR International Association, 2016). A controlled study of EMDR treatment with a comparison group for CBT with cancer patients illustrated that those who received EMDR treatment were significantly freer of PTSD symptoms at the conclusion of treatment compared with a CBT group (Capezzani et al., 2013). A case study using EMDR in a child's processing focused on the importance of a family systems approach although the child had a nonmalignant medical condition (Klaff, 2016). In an article outlining the role for EMDR therapy Faretta and Civilotti (2016) proposed that cancer-related traumas maintain a vicious cycle between psychological and physical health and that the application of EMDR therapy could break this cycle. A case study of a couple 
where one spouse had cancer integrated individual EMDR therapy for each spouse, along with conjoint therapy, to assist them in navigating the impact of cancer on the marriage (Moore, 2016). A pilot study compared standard EMDR therapy and CBT in the treatment of cancer survivors with diagnosed PTSD, who were in the active and follow-up phases of cancer treatment. Twenty-one patients were randomly assigned to one of the two treatments, with multiple measures administered at preintervention and at 1-month follow-up. At follow-up, the absence of a PTSD diagnosis was associated with a higher likelihood of having received EMDR therapy, whereas improvements in anxiety and depression were equal for both modalities (Capezzani et al., 2013).

Faretta, Borsato, Civilotti, Fernandez, and Pagani (2016) compared a nonmanualized supportive CBT approach and a nonstandard EMDR therapy protocol for oncology patients. This protocol specifically addressed crucial cancer-related traumatic memories, with the aim of restoring emotional and relational balance (Faretta \& Borsato, 2016). Fifty-seven participants were assigned to either EMDR therapy or nontrauma-focused CBT for a total of 12 sessions, and multiple measures were administered at pretreatment, midtreatment, and posttreatment. Results showed significant improvements on the majority of dependent variables with both modalities, but no improvement on any subscales of the Symptom Checklist-90-R for the CBT group (Faretta et al., 2016).

EMDR Group Treatment. The initial 11 years of EMDR therapy, the first wave, focused on individual EMDR interventions used in private practices and agencies. EMDR group therapies were then provided primarily in disaster settings with the use of the Integrative Group Treatment Protocol (IGTP) first introduced in 1998 (Jarero, Artigas, \& Hartung, 2006). Despite it being a relatively brief treatment, access to EMDR treatment may be limited when large numbers are in need, as existing mental health resources often have great difficulty in meeting requests for services. We believe that the EMDR group therapies herald the second wave for EMDR therapy, in which the access to effective EMDR treatment can be scaled up. Providing group therapies in various clinical settings will make economically viable psychosocial interventions more widely accessible everywhere.

The large number of cancer survivors who choose not to attend traditional support groups out of the concern they may be traumatized further reveals a need for alternative kinds of support that differ from those already available. EMDR group therapy differs from traditional group therapy in that the group members work independently on their own material. EMDR group protocols do not permit participants to verbalize their trauma narratives. These features are shared by both of the EMDR group protocols, the EMDR Group Traumatic Episode Protocol (G-TEP; Shapiro, 2013a), and the Integrative Group Treatment Protocol (IGTP; Jarero et al., 2016). The EMDR group protocols also afford flexibility, as their use does not require problem homogeneity. In this way, subjects avoid any ancillary triggers, bypass any possible social anxiety associated with speaking in a group, and may profit from some of the positive benefits that are common to a group experience (Yalom, 1970).

Related Research. Two seminal studies involved EMDR treatment of cancer patients using a group format, which was modified from the traditional EMDR IGTP (Jarero, Artigas, Montero, \& Lena, 2008). IGTP was adapted for adults and adolescents living with "Ongoing Traumatic Stress" and is referred to as the EMDR-IGTP-OTS (Jarero et al., 2015). A pilot study, evaluating its efficacy in reducing cancer-related PTSD symptoms in women, showed significant improvement in PTSD symptoms and subjective well-being. Twenty-four female patients with various kinds of cancer in the active and follow-up phases of cancer received the IGTP on three consecutive days twice daily. PTSD symptoms were measured using the Short Posttraumatic Stress Disorder Interview (SPRINT). ANOVA analysis showed significant main effects for time and group, and subjective improvements in trauma symptoms were also reported by the participants (Jarero et al., 2015).

This protocol was implemented in a single case study, effectively treating a patient with cancer (Jarero et al., 2016). The study illustrated the detailed procedures and the client's positive responses. Evidence from these recent studies indicates that EMDR group therapy may be an effective modality for the psychological treatment of cancer survivors.

\section{EMDR G-TEP}

The EMDR Group Traumatic Episode Protocol (G-TEP) is a new protocol developed by Shapiro and Laub with some similarities to the IGTP but also very distinctive differences. It was originally introduced in a 2013 presentation (Shapiro, 2013a). It is a group modification of the Recent Traumatic Episode Protocol (R-TEP), which was specifically designed for recent 


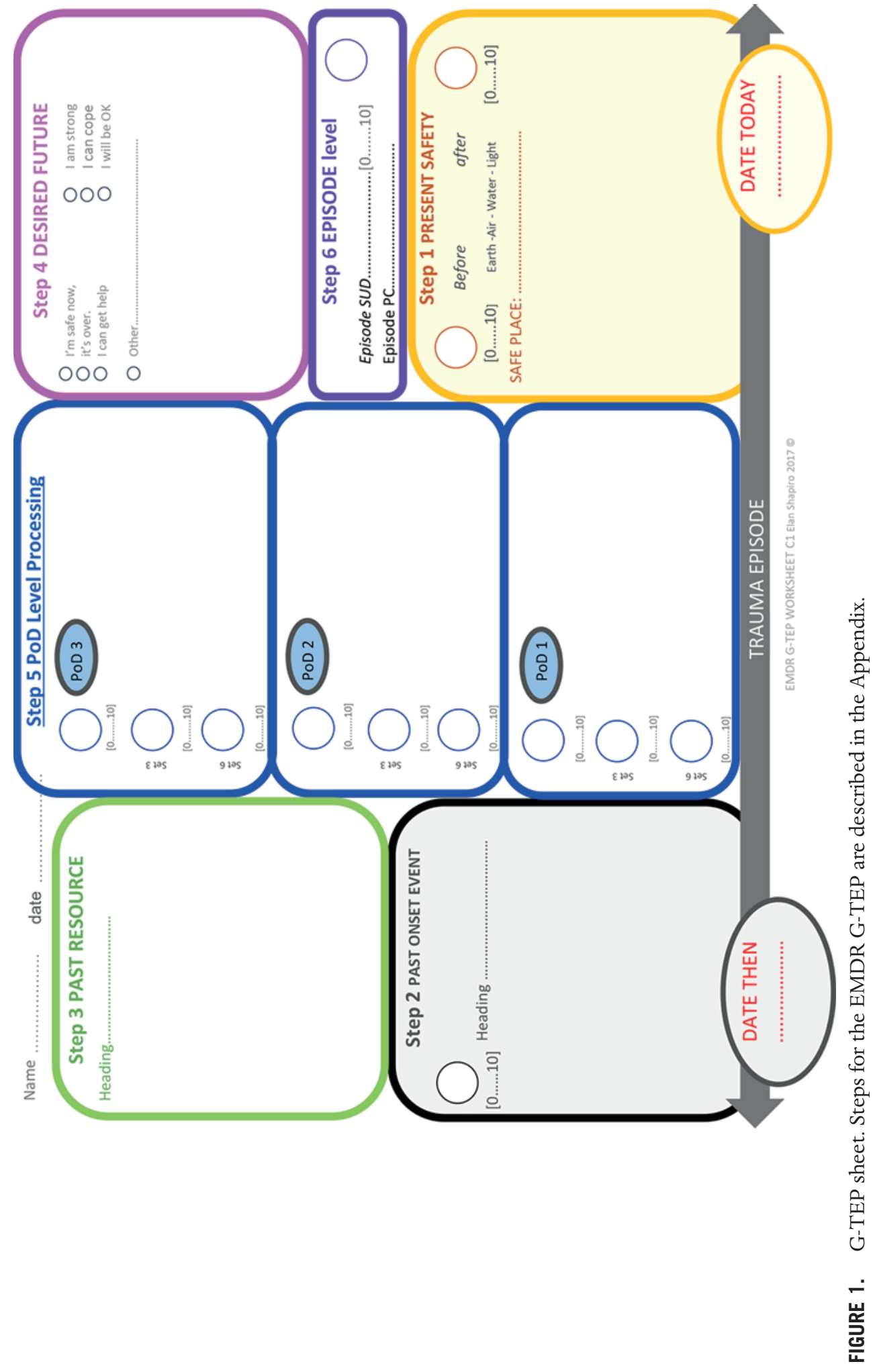


traumatic events with ongoing consequences and is therefore ideally suited to an oncology population (E. Shapiro \& Laub, 2008, 2015). EMDR G-TEP has been used following the terrorist attacks in Belgium, France, Great Britain, Turkey, and in various disaster zones in the United States, Europe, and Asia. Several studies are underway around the globe.

\section{Description of the EMDR G-TEP Protocol}

Each participant is seated at a table with the other group members and is given an EMDR G-TEP worksheet comprising four sections marked on the sheet that surround the central portion (see Figure 1). The central portions on the sheet symbolize the trauma material to be processed; three of the outer sections represent past, present, and future resources such as a safe place, a past positive memory situation, activity, or event, and a desired future. In the EMDR G-TEP, a central feature of the protocol is the utilization of an EMD-type strategy. EMD is the original procedure pioneered by Francine Shapiro which is primarily a desensitization procedure unlike the standard EMDR protocol. The processing is considered to be restricted, focusing on associations to the points of disturbance and confined only to content related to the point of disturbance, PoD (Shapiro, 1989). Participants are familiarized with the Subjective Units of Distress Scale (SUD), where zero represents no disturbance and 10, maximum disturbance (Shapiro, 2018; Wolpe, 1969). The use of the subjective units of disturbance (SUD) scale is part of EMDR therapy's standard procedure. It was adopted from behavior therapy, used to assess an individual's level of activation on a scale of $0-10$, where 0 is calm and 10 is the highest level of anxiety (Wolpe, 1969). Participants are instructed on how to use the self-administered bilateral stimulation (BLS) with the Butterfly Hug (Artigas \& Jarero, 2007; Jarero \& Artigas, 1999). During the trauma processing component of the EMDR G-TEP protocol, eye movements are performed by following one hand as it is moved back and forth, alternately tapping identified sections on the article. This is also the case when participants are identifying a PoD using the "Google search," which is borrowed from the individual version, the R-TEP (E. Shapiro, 2014). They are also instructed to write or draw representative material in the designated sections on the article. See Appendix for detailed steps.

\section{Research on EMDR G-TEP}

A randomized clinical trial with Syrian refugees in a Turkish refugee camp revealed posttraumatic stress disorder symptoms that were significantly decreased after two EMDR G-TEP sessions (Yurtsever et al., 2018). Twenty-nine individuals were randomly assigned to either the treatment group or a waitlist control group. Two EMDR G-TEP sessions were administered with pre, post, and follow-up measures using the Impact of Events Scale Revised (IES-R), the Beck Depression Inventory (BDI), and the Mini International Neuropsychiatric Inventory (MINI). The percentage of PTSD reduced from $100 \%$ to $38.9 \%$ in the treatment group and remained unchanged in the control. Sixty-one percent of the treatment group no longer satisfied diagnostic criteria for PTSD and results were maintained at 1-month follow-up.

A field study with traumatized refugees in Germany showed significant reductions in trauma and depression with the EMDR G-TEP protocol after only two 90-minute sessions (Lehnung, Shapiro, Schreiber, \& Hofmann, 2017). After receiving a psychoeducation session, 18 Arabic-speaking refugees were assigned to treatment or waitlist. Pre- and postmeasures were taken using the IES-R and BDI. Significant differences were observed between treatment and waitlist for IES-R scores $(p<.05)$ but not for BDI scores.

\section{The Current Study}

No reports to date reflect studies of the EMDR G-TEP protocol with a medical population, supporting the need for this preliminary research. The purpose of this pre-experimental case study was to explore the possible efficacy and safety of the EMDR G-TEP in the psychological treatment of cancer survivors. We hypothesized that EMDR G-TEP would significantly lower scores on three standardized measures evaluating the presence and severity of symptoms consistent with either depression, anxiety, or a trauma-related disorder.

\section{Method}

This research was a pre-experimental case study conducted in one site in Amherst, Massachusetts following IRB approval from the Asentral, Inc. Institutional Review Board (IRB) in Newburyport, Massachusetts.

\section{Participants}

Participants were recruited from a wide area throughout Massachusetts through hospital systems, cancer support groups, oncologists, primary care settings, mental health clinicians, newspaper advertising, public speaking presentations, community 
forums, and freestanding cancer organizations. All prospective participants meeting inclusion criteria were offered the opportunity to participate in the study. Inclusion criteria were: (a) an existing cancer diagnosis received within the past year; (b) undergoing cancer treatment within the past year; and (c) ability to physically, cognitively, and emotionally tolerate a trauma treatment. Persons meeting any exclusion criteria were excluded from the study. The exclusion criteria were as follows: (a) current, active chemical dependency problem; (b) active suicidal tendencies or engaging in self-harm; and (c) evidence of an active psychosis, mania, pregnancy, or an age of less than 18 years.

Following signed informed consent, prospective participants underwent an extensive 60- to 90-minute screening with the principal investigator using an IRB-approved intake interview format. Screening included a history taking and thorough assessment of current functioning to ensure tolerance to the trauma treatment. Components included the Karnofsky Performance Scale; the Functional Assessment for Cancer Patients, General Population (FACT-GP); a full Mental Status Exam; an orientation to EMDR Therapy; and a manual and video demonstration of the EMDR G-TEP protocol. Since chemotherapy is known to cause cognitive changes in many patients (Jansen, Cooper, Dodd, \& Miaskowski, 2011), the Mental Status Exam determined whether participants would be able to engage in the cognitive and somatic processing required in order to safely and effectively receive benefits from the EMDR therapy.

\section{Therapist, Clinician Assistants, and Research Assistants}

The principal investigator individually screened each potential participant and led the EMDR G-TEP sessions. She is an EMDR R-TEP, G-TEP trainer, EMDRIA-approved consultant, and doctoral level clinical psychologist with a specialty in behavioral medicine.

Clinical Assistants. Given the vulnerable population, specific measures were incorporated to ensure the safety and well-being of participants. The principal investigator gave 10 qualified, licensed EMDR therapy clinicians standardized instructions and training, specific to the study procedures, prior to commencement of the implementation phase of the study. Clinician assistants were only involved in the administration of the EMDR G-TEP intervention, monitoring, and management of subjects on the day of the treatment and/or testing, but not with any other aspect of the study. The clinical roles were as follows: (a) One participant monitor was assigned to attend to the well-being of participants during the EMDR G-TEP sessions. The monitor circulated the room while the protocol was administered and was available for questions and clarification and any additional assistance required. (b) One emotional protection therapist (EPT) was assigned to be available as a safety measure in the event any participants became acutely medically unwell or experienced any psychological abreactions. This clinician was stationed outside the treatment room as a precautionary measure. No serious adverse events occurred with any of the participants that required EPT involvement. Two participants verbally reported feeling increased emotional dysregulation after the first EMDR G-TEP session, which spontaneously resolved by the end of the second session the following day. (c) One EMDR G-TEP fidelity monitor per group session was given an EMDR G-TEP fidelity checklist (Shapiro \& Moench, 2015) to ensure standardization and adherence to the protocol, which was conducted and administered by the principal investigator. All ratings on the fidelity checklist were satisfactory. (d) One testing administrator was assigned per group and at the 1-month follow-up phase. The testing administrators administered the testing instruments to the research participants and collected the coded data independently of the principal investigator, who was not present in the room during testing.

Research Assistants. A trained administrative assistant performed data entry. An independent research consultant performed the statistical analyses. The study was single blind with coding of participant identities and all collected data. Data were collected, stored, and handled in full compliance with IRB requirements to ensure confidentiality, in accordance with state, federal, and privacy protection laws.

\section{Intervention}

The intervention was offered twice on different weekends. All participants received one 90-minute EMDR G-TEP session on two consecutive days on a single weekend. The treatment group and the delayed treatment group were administered 1 month apart. Participants were assigned at random to one of the two intervention conditions. No participants were assigned to a control group.

\section{Data Collection and Measures}

Participants were administered preintervention measures immediately prior to the first EMDR G-TEP intervention on day 1 , postintervention measures 
immediately following the second EMDR G-TEP session on day two, and at 1-month follow-up.

SPRINT. The Short Posttraumatic Stress Disorder Rating Interview (Connor $\&$ Davidson, 2001) consists of four items corresponding to each of the four PTSD symptom clusters. Acceptable test-retest reliability was established with a value of $0.778(p<.001)$ (Connor \& Davidson, 2001). The instrument also has good interrater reliability (Connor $\&$ Davidson, 2001). The convergent validity assessed with other instruments was acceptable (Connor \& Davidson, 2001). The SPRINT, therefore, demonstrates acceptable psychometric properties and can serve as a reliable validindicator of PTSD illness severity and of global improvement. On average, the SPRINT takes 5-10 minutes to complete and is administered easily, thus not unduly burdening subjects.

STAI. The State-Trait Anxiety Inventory (STAI, adult version) is a self-report instrument that measures the presence and severity of current symptoms of anxiety and a generalized propensity to be anxious (Spielberger, 2016). The STAI measures state and trait anxiety, which is of particular interest given the pre- and posttest measurements occurred within 24 hours and then 30 days after the last intervention. The intent was to examine data on trait change at the 30-day mark and any significant changes in state anxiety compared to the earlier administrations. The STAI consists of two subscales. The State Anxiety Scale (S-Anxiety) evaluates the current state of anxiety. The Trait Anxiety Scale (T-Anxiety) evaluates relatively stable aspects of anxiety proneness. The STAI has 40 items, with 20 items allocated to each of the two subscales. Test-retest reliability coefficients ranged from 0.31 to 0.86 on initial development. The STAI was validated with more than 10,000 adults during its development. This measure is brief to administer and does not require costly or time-consuming scoring or interpretation procedures. It is, therefore, appropriate for research with a medical population.

BDI. The BDI-II has been increasingly used with the medically ill to evaluate depressive states that occur at high prevalence in healthcare settings (Beck, Steer, \& Brown, 1996). The BDI-II is a brief scale that is acceptable to participants and clinicians, covers all the Diagnostic and Statistical Manual of Mental Disorders (5th ed.; DSM-5; American Psychiatric Association, 2013) diagnostic criteria for major depressive disorder, and stands as a reliable indicator of symptom severity and suicidal thoughts. The BDI-II has well established reliability, validity, and case finding capability as a screening instrument.

\section{Data Analysis}

The analysis focused on examining within group differences over time for the combined sample (two intervention groups) on multiple measures, three of which were used to evaluate the presence and severity of symptoms consistent with either depression, anxiety, or a trauma-related disorder, SPRINT, STAI S-Anxiety, STAI T-Anxiety, and BDI-II. The distribution of the data for each dependent variable was assessed for normality via skewness and kurtosis. Repeated measures of Analysis of Variance (ANOVA) and paired comparisons with a Bonferroni correction served to examine within-group differences from administration to administration between the pretest, the posttest, and the 1-month follow-up. Effect sizes were calculated using partial $\eta^{2}$ for ANOVA and Cohen's $d$ for paired comparisons. The small sample size precluded the ability to conduct multivariate analyses with adequate statistical power.

\section{Results}

\section{Sample Characteristics}

More than three quarters of the participants were women (see Table 1). More than half of the participants were married. The effects of marital status on survival of cancer survivors was examined. Unmarried persons with cancer had decreased overall

\section{TABLE 1. Participant Characteristics}

\begin{tabular}{llc}
\hline & & $n(\%)$ \\
\hline \multirow{2}{*}{ Mender } & Male & $8(22.9)$ \\
& Female & $27(77.1)$ \\
& Married status & $19(54.3)$ \\
& Single & $7(20.0)$ \\
& Divorced & $2(5.7)$ \\
Education attained & High school & $4(11.4)$ \\
& Training/apprentice & $3(8.6)$ \\
& Associate degree/ & $3(8.6)$ \\
& diploma & $4(11.4)$ \\
& Bachelor degree & $6(17.1)$ \\
& Graduate & $15(42.9)$ \\
Ethnicity & Missing & $5(14.3)$ \\
& White & $35(100.0)$ \\
\hline
\end{tabular}


survival (Goodwin, Hunt, Key, \& Samet, 1987). Fiftyfour individuals were screened and 35 were accepted into the study. Twenty-nine either did not meet inclusion criteria, chose not to participate, had scheduling conflicts, or elected not to participate for a variety of ongoing family and medical stressors. Despite efforts to recruit a diverse sample by making comprehensive overtures to major hospitals, clinics, and oncology practices in the area and removing all financial barriers for disadvantaged groups, all participants in the study were White. Only one African American indicated interest, was screened, and accepted, but failed to show for the intervention. Educational attainment was high, with $60 \%$ of the participants reporting having earned a bachelor degree or higher. The participants' age ranged from 29 to 84 . Most were middleaged or older $(M=62.9, S D=11.14)$. The median age was 64 and the mode was 60 . The majority were not experiencing a recurrence. There was one individual who arrived for the intervention but could not participate for medical reasons. Otherwise, there was $100 \%$ compliance.

The analysis focused on examining differences over time across the combined sample of 35 participants in the two intervention groups on multiple measures: SPRINT, STAI S-Anxiety, STAI T-Anxiety, and BDI-II.

\section{Descriptive Statistics}

Descriptive statistics for the dependent variables are presented in this section. The values for the three psychological measures appear in Table 2.

Measures of PTSD Symptoms, Anxiety, and Depression. Three standardized measures were used to evaluate the presence and severity of symptoms consistent with either a trauma-related disorder, state and trait

TABLE 2. Mean Scores and Standard Deviations on the SPRINT, STAI, and BDI-II

\begin{tabular}{lccc}
\hline & \multicolumn{3}{c}{ Combined Sample } \\
\cline { 2 - 4 } & Pre & Post & Follow-Up \\
\hline $\begin{array}{l}\text { SPRINT } \\
(n=35)\end{array}$ & $13.7(5.7)$ & $10.1^{\star}(6.2)$ & $9.3^{\star \star \star}(6.3)$ \\
$\begin{array}{l}\text { STAI-State } \\
(n=35)\end{array}$ & $43.4(13.5)$ & $36.7^{\star}(13.3)$ & $39.3(14.6)$ \\
$\begin{array}{l}\text { STAI-Trait } \\
(n=35)\end{array}$ & $46.5(13.0)$ & $40.4^{\star \star \star}(13.2)$ & $41.2^{\star}(14.3)$ \\
$\begin{array}{l}\text { BDI-II } \\
(n=33)\end{array}$ & $16.4(10.0)$ & $12.7^{\star \star \star}(10.2)$ & $10.0^{\star \star}(6.6)$ \\
\hline
\end{tabular}

Note. Comparison with pretreatment score.

${ }^{\star} p<.05 .{ }^{\star \star} p<.01 .{ }^{\star \star \star} p<.001$. anxiety, or depression. The mean score and standard deviation for each measure for the combined sample (two intervention groups) are presented in Table 2.

We also assessed the proportion of participants whose scores were above the clinical cutoff scores for the three measures. Connor and Davidson (2001) suggested a clinical cutoff for the SPRINT of 14, which we used. On the pretest, $54 \%$ of the participants scored above the cutoff score of 14, decreasing to $29 \%$ at posttest and $26 \%$ at follow-up. The clinical cutoff scores for the STAI scales are $39-40$ or $54-55$ for an older population. Given that most in this sample were middle-aged or older, we used a cutoff score of $54-55$. On the S-Anxiety pretest, $20 \%$ scored above the cutoff score of $54-55$, decreasing to $11 \%$ at posttest and $14 \%$ at follow-up. On the T-Anxiety pretest, $34 \%$ scored above the cutoff score of 54-55, decreasing to $14 \%$ at posttest and $20 \%$ at follow-up.

The clinical cutoff scores for the BDI-II are as follows: minimal depression (0-13), mild depression (14-19), moderate depression (20-28), and severe (29-63).

\section{Repeated Measures Comparisons}

SPRINT Comparisons Over Time. The SPRINT scores used in the analyses were the sum of Q1-Q8 scores. A repeated measures ANOVA revealed that mean SPRINT scores differed significantly between time points, $F(1,34)=24.146, p<.001, \eta_{p}{ }^{2}=0.417$. Post hoc tests using the Bonferroni correction revealed that two pairwise comparisons were significant. SPRINT scores were significantly higher at pretest $(M$ $=13.7)$ than posttest $(M=10.0), p=.04, d=.59$ and follow-up $(M=9.4), p<.001, d=.736$. No significant difference was found between scores on the SPRINT posttest and at follow-up.

STAI Comparisons Over Time. A repeated measures ANOVA determined that mean STAI scores differed significantly between time points for S-Anxiety, $F(1$, 34) $=6.114, p=.019, \eta_{\mathrm{p}}{ }^{2}=0.217$. Post hoc tests using the Bonferroni correction revealed one significant pairwise comparison for S-Anxiety. S-Anxiety scores were significantly higher for the pretest $(M=43.4)$ than for the posttest $(M=36.7), p<.001, d=.489$. No significant differences were found between S-Anxiety scores on the pretest and follow-up, or between the posttest and follow-up.

A repeated measures ANOVA determined that mean STAI scores also differed significantly between time points for T-Anxiety, $F(1,34)=9.460, p=.004$, $\eta_{\mathrm{p}}^{2}=0.223$. Post hoc tests using the Bonferroni correction revealed two significant pairwise comparisons for T-Anxiety. T-Anxiety scores were significantly 
higher for the pretest $(M=46.5)$ than for the posttest $(M=40.4), p<.001, d=.465$; and for the follow-up $(M=41.2), p=.013, d=.393$. However, there was no significant difference between T-Anxiety scores on the posttest and follow-up.

BDI-II Comparisons Over Time. A repeated measures ANOVA determined that mean BDI-II scores also differed significantly between time points, $F(1,32)=27.617, p<.001, \eta_{\mathrm{p}}{ }^{2}=0.463$. Post hoc tests using the Bonferroni correction revealed two significant pairwise comparisons for the scores on the BDI-II. Scores were significantly higher across the sample for the BDI-II pretest $(M=16.4)$ compared to the posttest $(M=12.67), p<.001, d=.369$ and compared to follow-up $(M=10.0), p=.004, d=.423$. No significant difference was found between BDI-II scores on the posttest and follow-up.

\section{Discussion}

Our preliminary hypothesis was that the EMDR G-TEP would significantly lower scores on three standardized measures evaluating the presence and severity of symptoms consistent with either depression, anxiety, or a trauma-related disorder. Repeated measures comparisons of measures of PTSD symptoms, anxiety, and depression revealed some significant differences over time. These results should be interpreted with caution in this pre-experimental case study, given the small sample size, the lack of randomization, the absence of a control group, and the possible influence of outliers on the mean scores. The inclusion criteria did not require a diagnosis of posttraumatic stress disorder, anxiety, or depression, and some participants' symptoms may not have been severe.

\section{Reductions in PTSD Symptoms}

PTSD symptom scores were significantly higher before treatment than at posttest and follow-up. This outcome is consistent with those produced in other EMDR-related treatments for cancer patients (Capezzani et al., 2013; Faretta et al., 2016; Jarero et al., 2013).

The incidence of posttraumatic stress disorder in cancer survivors is common. A recent study in Malaysia of 469 patients with various types of cancer revealed $21.7 \%$ suffered from posttraumatic stress disorder at 6 months postdiagnosis (Chan et al., 2018). At 4-year follow-up, prevalence had dropped to $6.1 \%$, indicating that an estimated $33 \%$ of those patients diagnosed with PTSD were still experiencing persistent symptoms - in many cases worsening - at the time of the 4-year follow-up.
It is striking that in this EMDR G-TEP study $54 \%$ of participants were above the SPRINT clinical cutoff at pretest. It is notable that at follow-up testing there was a reduction to an encouraging $26 \%$. This strongly suggests that psychological interventions such as EMDR G-TEP should not only be confined and focused on early intervention, but provided throughout the continuum of care, as in this study.

\section{Reductions in Depressive Symptoms}

The Beck Depression Inventory (BDI-II) has been used extensively with medical populations (Beck et al., 1996). Scores may be elevated due to symptoms related to the medical diagnosis and not depressive symptoms, although in this study participants serve as their own controls. Depressive symptoms can co-occur with serious medical illness. BDI-II scores were significantly higher on the pretest (compared to the posttest and follow-up). Effect sizes were only small-medium at posttreatment $(d=.369)$ and medium at follow-up $(d=.423)$. This finding is not consistent with those of Capezzani et al. (2013) or Faretta et al. (2016) and may be related to our participants reporting only mild depression at pretreatment.

However, it is very possible that participants receiving cancer treatment were suffering from treatment or illness-related symptoms that resemble depression and which are measured on the BDI-II as low energy, fatigue, changes in appetite, and changes in sleep. It has been asserted that depression measures that include somatic symptoms in a medically ill population may artificially inflate severity measures, thus creating bias. One study contradicts these assertions in a cardiac population (Thombs et al., 2010).

\section{Reductions in Symptoms of Anxiety}

State-Anxiety scores were significantly lower at posttest than pretest, but the results were not maintained at follow-up. In contrast, the trait-anxiety scores were significantly lower than pretest at follow-up.

The follow-up scores for trait anxiety (STAI T-Anxiety) were especially intriguing. Scores indicated a change in baseline anxiety, an assessment of personal response style, not merely symptomatic reduction (S-Anxiety). This suggests that there may have been a moderate change in participants' view of self with treatment. Changes in trait-anxiety following the EMDR therapy were examined and compared with standard care in an HMO setting yielding superior results (Marcus, Marquis, \& Sakai, 1997). The current study findings suggest that the EMDR G-TEP may be 
effective in reducing both situational anxiety and baseline trait anxiety.

\section{Length of Treatment}

Changes between posttest and follow-up across the entire sample were modest. Two EMDR G-TEP sessions were a minimal level of intervention and may possibly indicate that dosing was insufficient, rather than attributing these modest changes to small sample size, and yet, the results are promising. In related research, Jarero et al. (2015) provided EMDR-IGTP-OTS to a sample of 24 women with cancer diagnoses, providing six group sessions. SPRINT scores for these participants reduced from 16.32 (5.04) at pretreatment to 5.41 (2.87) at posttreatment and 3.73 (3.99) at 90-day follow-up.

\section{Limitations}

The use of a pre-experimental case study design precludes the ability to infer that participation in the EMDR G-TEP protocol caused these significant differences due to the inability to isolate the effect of the treatment due to the lack of a control group. The results support the need for further research using an experimental pretest-posttest control group design with a large sample to examine the efficacy of EMDR G-TEP in reducing psychological symptoms following a cancer diagnosis.

Due to the constraints of a private practice setting and the limitations of available volunteer clinicians, the principal investigator performed both the initial screening interview and the EMDR G-TEP intervention. This dual role limits the study by introducing possible researcher bias. However, the screening was structured and uniform; inclusion/exclusion criteria were standardized to minimize the impact of bias and the implementation of the group protocol strictly monitored by fidelity raters.

A 1-month follow-up is a limitation. A 3-month and 1-year follow-up would have been optimal; however, due to the high probability of attrition and incomplete data and the more important priority of gathering preliminary data about the EMDR G-TEP with cancer survivors, this option was not pursued. We also chose a modest number of the EMDR G-TEP sessions and minimal time commitment, in part to minimize the burden on participants.

\section{Implications for Practice}

The nature of pre-experimental research limits the implications for practice. Results from a large-scale experimental study may reveal the effect of the EMDR
G-TEP on PTSD, anxiety, and depression on oncology patients. If further research demonstrates the efficacy of the EMDR G-TEP, those findings may have implications for other medical populations. Furthermore, problem homogeneity within the EMDR G-TEP group is not a necessary condition for its effectiveness. Participants can be working on completely different issues to their peers and still obtain robust changes.

\section{Recommendations and Future Directions for Research}

The results support the need for further research using an experimental pretest-posttest control group design with a large sample to examine the efficacy of EMDR G-TEP in reducing psychological symptoms post-cancer diagnosis. Conducting the study at regional cancer hubs may provide access to a large sample of oncology patients from which to select and assign participants randomly to treatment and control groups. When feasible, principal investigators should not be involved in the dual role of recruitment and provision of the intervention to avoid potentially biased responses.

Offering more than two EMDR G-TEP sessions might lead to more robust results, assuming a dose-related response. Further studies examining the results of a larger dose effect of EMDR G-TEP sessions would be intriguing.

If further research generates evidence of the efficacy of the EMDR G-TEP as a stand-alone intervention, then a worthwhile follow-up comparative study could examine the relative effectiveness of the EMDR G-TEP against other modalities, such as the IGTP and CBT trauma-focused group protocols for cancer survivors.

\section{Conclusions}

Cancer is a chronic, sometimes life-threatening disease with ongoing consequences, accompanied by the constant threat of recurrence, unlike a discrete single event trauma with a definite endpoint where a complete resolution is possible. Therefore, a cancer diagnosis is consistent with expectations for ongoing traumatic stress. Reduction in distress and psychological symptoms with the EMDR G-TEP may assist the cancer survivor in managing the ongoing challenges of often brutal medical regimens, lifestyle adjustments, social-emotional difficulties, occupational stress or loss of ability to work, and in the promotion of overall resilience. 


\section{References}

American Psychiatric Association. (2013). Diagnostic and statistical manual of mental disorders (5th ed.). Arlington, VA: American Psychiatric Publishing.

Andrykowski, M., \& Kangas, M. (2010). Posttraumatic stress disorder associated with cancer diagnosis and treatment. In J. C. Holland, W. S. Brietbart, P. B. Jacobsen, M. S. Lederberg, M. J. Loscalzo, \& R. McCorklen (Eds.), Psycho-oncology (Vol. 2, pp. 348-357). New York, NY: Oxford University Press.

Artigas, L., \& Jarero, I. (2007). The butterfly hug. Mexico City, Mexico: Asociación Mexicana para Ayuda Mental en Crisis.

Artigas, L., \& Jarero, I. (2014). The butterfly hug. In M. Luber (Ed.), Implementing EMDR early interventions for man-made disasters and natural disasters: Models scripted protocols, and summary sheets (pp. 127-131). New York, NY: Springer Publishing.

Beck, A. T., Steer, R. A., \& Brown, G. (1996). Beck Depression Inventory (BDI-II) (2nd ed.). Boston, MA: Pearson.

Capezzani, L., Ostacoli, L., Cavallo, M., Carletto, S., Fernandez, I., Solomon, R., . . .Cantelmi, T. (2013). EMDR and CBT for cancer patients: Comparative study of effects on PTSD, anxiety and depression. Journal of EMDR Practice and Research, 7(3), 134-143. http://dx. doi.org/10.1891/1933-3196.7.3.134

Chan, C. M. H., Ng, C. G., Taib, N. A., Wee, L. H., Krupat, E., \& Meyer, F. (2018). Course and predictors of post-traumatic stress disorder in a cohort of psychologically distressed patients with cancer: A 4-year follow-up study. Cancer, 124(2), 406-416. http://dx.doi.org/10. 1002 / cncr.30980

Connor, K. M., \& Davidson, J. R. (2001). SPRINT: A brief global assessment of post-traumatic stress disorder. International Clinical Psychopharmacology, 16(5), 279-284. http: / / dx.doi.org/10.1097 / 00004850-200109000-00005

EMDR International Association. (2012). Definition of EMDR. Retrieved from http://c.ymcdn.com/sites/ www.emdria.org/resource/resmgr/Definition/EMDR IADefinitionofEMDR.pdf

EMDR International Association. (2016). Special issue on EMDR and psycho-oncology. Journal of EMDR Practice and Research, 10(3).

Faretta, E., \& Borsato, T. (2016a). EMDR therapy protocol for oncology patients. Journal of EMDR Practice and Research, 10(3), 162-175. http://dx.doi.org/10.1891/ 1933-3196.10.3.162

Faretta, E., Borsato, T., Civilotti, C., Fernandez, I., \& Pagani, M. (2016). EMDR and CBT: A comparative clinical Study with oncology patients. Journal of EMDR Practice and Research, 10(3), 215-227. http://dx.doi.org/10. 1891/1933-3196.10.3.215

Faretta, E., \& Civilotti, C. (2016c). EMDR therapy in psycho-oncology: A bridge between mind and body. Journal of EMDR Practice and Research, 10(3), 138-152. http: / / dx.doi.org/ 10.1891/1933-3196.10.3.138
Galinsky, M. J., \& Schopler, J. H. (1994). Negative experiences in support groups. Social Work in Healthcare, 20(1), 77-95. http: / / dx.doi.org/10.1300/J010v20n01_09

Giese-Davis, J., Brandelli, Y., Kronenwetter, C., Golant, M., Cordova, M., Twirbutt, S., . . .Spiegel, D. (2016). Illustrating the multi-faceted dimensions of group therapy and support for cancer patients. Healthcare, 4(3), 48-61. http: / / dx.doi.org/10.3390/ healthcare4030048

Goodwin, J. S., Hunt, W. C., Key, C. R., \& Samet, J. M. (1987). The effect of marital status on stage, treatment, and survival of cancer patients. JAMA: The Journal of the American Medical Association, 258(21), 3125-3130. http: / / dx.doi.org/10.1001/jama.1987.03400210067027

Hoeck, B., Ledderer, L., \& Hansen, H. P. (2015). Involvement of patients with lung and gynecological cancer and their relatives in psychosocial cancer rehabilitation: A narrative review. The Patient-Centered Outcomes Research, 8(2), 127-143. http://dx.doi.org/10.1007/ s40271-014-0076-x

House, J., Landis, K., \& Umberson, D. (1988). Social relationships and health. Science, 241(4865), 540-545. http: / / dx.doi.org/10.1126/science.3399889

Jansen, C. E., Cooper, B. A., Dodd, M. J., \& Miaskowski, C. A. (2011). A prospective longitudinal study of chemotherapy-induced cognitive changes in breast cancer patients. Supportive Care in Cancer, 19(10), 1647-1656. http: / / dx.doi.org/10.1007/ s00520-010-0997-4

Jarero, I., Amaya, C., Givaudan, M., \& Miranda, A. (2013). EMDR individual protocol for paraprofessional use: A randomized controlled study for first responders. Journal of EMDR Practice and Research, 7(2), 55-64. http://dx. doi.org/10.1891/1933-3196.7.2.55

Jarero, I., \& Artigas, L. (1999). The butterfly hug. EMDRIA Newsletter, 4(4), 11-13.

Jarero, I., Artigas, L., \& Hartung, J. (2006). EMDR integrative group treatment protocol: A postdisaster trauma intervention for children and adults. Traumatology, 12(2), 121-129. http: / dx.doi.org/10.1177/1534765606294561

Jarero, I., Artigas, L., \& Luber, M. (2011). The EMDR protocol for recent critical incidents: Application in a disaster mental health continuum of care context. Journal of EMDR Practice and Research, 5(3), 82-94. http: / / dx.doi.org/10.1891/1933-3196.5.3.82

Jarero, I., Artigas, L., Montero, M., \& Lena, L. (2008). The EMDR integrative group treatment protocol: Application with child victims of a mass disaster. Journal of EMDR Practice and Research, 2(2), 97-105. http: / / dx.doi. org/10.1891/1933-3196.2.2.97

Jarero, I., Artigas, L., Uribe, S., Garcia, L. E., Cavazos, M. A., \& Givaudan, M. (2015). Pilot research study on the provision of the EMDR integrative group treatment protocol with female cancer patients. Journal of EMDR Practice and Research, 9(2), 98-105.

Jarero, I., Artigas, L., Uribe, S., \& García, L. E. (2016). The EMDR integrative group treatment protocol for patients with cancer. Journal of EMDR Practice and Research, 10(3), 199-207. http: / / dx.doi.org/ 10.1891 / 1933-3196.10.3.199 
Klaff, F. (2016). EMDR therapy as portal to a child's processing of a medical condition: A case study and session deconstruction. Journal of EMDR Practice and Research, 10(3), 189-198. http://dx.doi.org/10.1891/ 1933-3196.10.3.189

Lehnung, M., Shapiro, E., Schreiber, M., \& Hofmann, A. (2017). Evaluating the EMDR Group traumatic episode protocol with refugees: A field study. Journal of EMDR Practice and Research, 11(3), 129-138. http:/ / dx.doi.org/ 10.1891/1933-3196.11.3.129

Marcus, S. V., Marquis, P., \& Sakai, C. (1997). Controlled study of treatment of PTSD using EMDR in an HMO setting. Psychotherapy: Theory, Research, Practice. Training, 34(3), 307-315.

Mariotto, A. B., Robin Yabroff, K., Shao, Y., Feuer, E. J., \& Brown, M. L. (2011). Projections of the cost of cancer care in the United States: 2010-2020. JNCI Journal of the National Cancer Institute, 103(2), 117-128. http: / / dx.doi. org/10.1093/jnci/djq495

Moore, M. V. (2016). Couple therapy when one spouse has cancer: Integration of EMDR and relationship enhancement therapies. Journal of EMDR Practice and Research, 10(3), 208-214. http://dx.doi.org/10.1891/1933-3196. 10.3.208

Morasso, G., Di Leo, S., Grassi, L., Amadori, M. L., Bellani, P., Bruzzi, P. G., \& Orru, W. (2002). La psiconcologia: Stato dell'arte. In Psiconcologia. Milan, Italy: Masson.

Naughton, M. J., \& Weaver, K. E. (2014). Physical and mental health among cancer survivors: Considerations for long-term care and quality of life. North Carolina Medical Journal, 75(4), 283-286.

Roberts, A. K. P. (1993) The effect of laughter and humor on the quality of life with cancer patients. Unpublished doctoral dissertation, University of Michigan, Ann Arbor, Michigan.

Shapiro, E., \& Moench, J. (2015). EMDR G-TEP fidelity scale for the group traumatic episode protocol. Retrieved from https://emdrresearchfoundation.org/ toolkit/gtep.pdf

Shapiro, E. (2013a). "The EMDR Group Traumatic Episode Protocol" Presentation to the EMDR Turkey Conference, Istanbul, Turkey.

Shapiro, F. (2013b). The case: Treating Jared through eye movement desensitization and reprocessing therapy. Journal of Clinical Psychology, 69(5), 494-496. http:/ / dx. doi.org/10.1002/jclp.21986

Shapiro, E., \& Laub, B. (2008). Early EMDR intervention (EEI): A summary, a theoretical model, and the recent traumatic episode protocol (R-TEP). Journal of EMDR Practice and Research, 2(2), 79-96. http:/ / dx.doi.org/10. 1891/1933-3196.2.2.79

Shapiro, E., \& Laub, B. (2015). Early EMDR intervention following a critical community incident: A randomized clinical trial. Journal of EMDR Practice and Research, 9(1), 17-27. http: / / dx.doi.org/10.1891/ 1933-3196.9.1.17

Shapiro, E. (2014). The recent episode protocol (R-TEP): An integrative protocol for early EMDR intervention (EEI).
In M. Luber (Ed.), Implementing EMDR early mental health interventions for man-made and natural disasters: Models, scripted protocols and summary sheets (pp. 193-207). New York, NY: Springer Publishing.

Shapiro, F. (2012). Getting past your past. Emmaus, PA: Rodale, Inc.

Shapiro, F. (2004). Theory: The adaptive information processing model. Watsonville, CA: EMDR Institute, Inc.

Shapiro, F. (2018). Eye movement desensitization and reprocessing (EMDR) therapy: Basic principles, protocols and procedures (3rd ed). New York, NY: Guilford Press.

Shapiro, F. (2001). Eye movement desensitization and reprocessing (EMDR) therapy: Basic principles, protocols, and procedures (2nd ed.). New York, NY: Guilford Press.

Shapiro, F. (1989). Efficacy of the eye movement desensitization procedure in the treatment of traumatic memories. Journal of Traumatic Stress, 2(2), 199-223. http://dx.doi. org/ $10.1002 /$ jts. 2490020207

Solomon, R. M., \& Shapiro, F. (2008). EMDR and the adaptive information processing model: Potential mechanisms of change. Journal of EMDR Practice and Research, 2(4), 315325. http: / / dx.doi.org/10.1891/1933-3196.2.4.315

Spiegel, D., Bloom, J. R., Kraemer, H. C., \& Gottheil, E. (1989). Effect of psychosocial treatment on survival of patients with metastatic breast cancer. The Lancet, 2(8668), 888-891. http: / / dx.doi.org/10.1016/S0140-6736(89)91551-1

Spielberger, C. D. (2016). The state-trait anxiety inventory [Manual]. Retrieved from http://www.mindgarden. com/145-state-trait-anxiety-inventory-for-adults

Thombs, B. D., Ziegelstein, R. C., Pilote, L., Dozois, D. J., Beck, A. T., Dobson, K. S., . . Abbey, S. E. (2010). Somatic symptom overlap in beck depression Inventory-II scores following myocardial infarction. British Journal of Psychiatry, 197(1), 61-65. http:/ / dx.doi.org/10.1192/bjp.bp.109. 076596

Wolpe, J. (1969). Practice of behavior therapy. New York, NY: Pergamon Press.

World Health Organization. (2013). New clinical protocol and guidelines to enable effective mental health care for adults and children exposed to trauma and loss. Retrieved from http://www.who.int/mediacentre/news/releases/ 2013/trauma_mental_health_20130806/en

World Health Organization. (2017). Cancer fact sheet 2017. Retrieved from http://www.who.int/mediacentre/ factsheets / fs297/en/

Yalom, I. D. (1970). The theory and practice of group psychotherapy (15th ed.). New York, NY: Basic Books.

Yurtsever, A., Konuk, E., Akyüz, T., Zat, Z., Tükel, F., Çetinkaya, M., . . Shapiro, E. (2018). An eye movement desensitization and reprocessing (EMDR) group intervention for syrian refugees with post traumatic stress symptoms. Frontiers in Psychology, 9, 493. http:/ / dx.doi.org/10. 3389/fpsyg.2018.00493, June

Correspondence regarding this article should be directed to Amanda Karen Patricia Roberts, PhD, MA, Private Practice, Amherst, MA 01001. E-mail: britshrink@aol.com 


\section{APPENDIX}

\section{Summary of the Procedures for the EMDR G-TEP}

Phase 1: History Taking

A careful individual screening intake interview is conducted.

Phase 2: Preparation, Step 1

Step 1 of the EMDR G-TEP involves a self-regulation exercise called the Four Elements (E. Shapiro, 2007). This exercise includes grounding, breathing, producing saliva, and accessing a safe place while engaging in bilateral self-administered Butterfly Hugs (BH; Artigas \& Jarero, 2014). This exercise serves not only as a stabilization preparation technique but also as a further screening. Those who are not able to reduce their SUD score by at least one point are not considered to be appropriate candidates for trauma processing. During Phase 2 participants are also directed to develop a safe place that they visualize, then write, or draw, in a designated section followed by self-administered bilateral stimulation (BLS; Shapiro, 2018). Participants are invited to verbally share their safe place with others in the group.

Phase 3: Assessment, Steps 2, 3, and 4

Step 2: Participants identify the onset traumatic event and rate their distress level 0 to 10 , which is written on the page, and are invited to draw or write a representation in the designated section. The negative cognition, validity of cognition (VOC), and body scan are omitted in this protocol. Step 3: Past resource. Participants access a memory of a time in their life when they felt really good about themselves. They are invited to draw or write a representation in the designated section. This is strengthened and installed with the Butterfly Hug. Participants are invited to verbally share with others in the group without pressure to do so if they choose not to.

Step 4: Desired future. A positive cognition is identified with the aid of a checklist as well as an open option. Participants are invited to draw or write a representation in the designated section. They are invited to verbally share their desired future.
Phase 4: Desensitization, Steps 4 and 5

A mental scan or metaphorical "Google search" of the trauma episode is performed by moving a hand laterally from one side of the EMDR G-TEP sheet (tapping the "Date Today" circle) to the other side (tapping the "Date Then" circle) while following with the eyes, until a Point of Disturbance (PoD) is identified and its SUD rating is recorded. In each session three target fragments or PoDs are identified in this manner, one at a time. Step 5: After a PoD is identified, an EMD type strategy is employed for each PoD, utilizing a total of nine sets of eye movements (EM) per PoD, returning to focus on the target PoD after every three sets and checking the SUD. In all, four SUD measurements are taken for each POD. The participants are instructed to place a hand on the PoD they are working on and follow their hand with their eyes as they alternately touch the step 1 section on the EMDR G-TEP sheet and the PoD section. The group leader tracks and audibly counts the number of sets to help establish group cohesion, dual attention focus essential to the efficacy of EMDR therapy processing, and additional grounding.

\section{Phase 5: Installation, Step 6}

Participants are asked to rate their level of distress as they think of the whole trauma episode and give a global SUD rating. A final episode positive cognition is selected after group sharing. It is installed using the Butterfly Hug technique, while participants are asked to hold the positive cognition in mind along with the entire episode.

Phase 6: Body Scan

No body scan is performed in EMDR G-TEP.

\section{Phase 7: Closure}

Closure is completed with the Four Elements Exercise as described previously in Phase 2. An informal debriefing of the whole group experience follows for approximately $20-30$ minutes.

\section{Phase 8: Re-Evaluation and Follow-Up}

Re-evaluation is performed on follow-up. Note that to competently and safely deliver this protocol the full training/consultation should be obtained from qualified practitioners. 